\title{
Face Recognition System Based on the Second Generation Identity Card
}

\author{
Xin $\mathrm{Ni}^{1,2}$, Zhen Lei ${ }^{1}$, Hui Xing ${ }^{1,2}$, Dong $\mathrm{Yi}^{1}$ \\ ${ }^{1)}$ Institute of Automation, Chinese Academy of Sciences, Beijing, China (xin.ni@ foxmail.com) \\ ${ }^{2)}$ Research and Development Center for Internet of Things, Chinese Academy of Sciences, Wuxi, Jiangsu, China
}

\begin{abstract}
In this paper, we develop a new face recognition system that was designed to use the second generation identity card (2G-ID card). In order to ensure the security in enrollment, the new subject is registered in a verification way. In enrollment phase, the captured image is firstly compared with the face image stored in 2G-ID card. If the similarity between these images is greater than a pre-defined threshold, the face image is registered to the system with the information from the 2G-ID card. In query phase, the captured image is compared to the templates stored in the database of the system and the top $\mathbf{N}$ similar face images are returned. Moreover, we can also use the additional information such as name and the ID number to assist the query. Experimental results validate that the quality assessment of face images and the custom design of communication packet format can improve the efficiency and accuracy of the system.
\end{abstract}

Keywords-2G-ID card, face recognition, enrollment, quality assessment, query

\section{基于二代证身份信息的人脸识别系统 \\ 倪釒金 $^{1,2}$ 雷震 $^{1} \quad$ 邢辉 ${ }^{1,2}$ 易东 $^{1}$ \\ 1) 中国科学院自动化研究所, 北京, 中国 \\ 2) 中国科学院物联网研究发展中心，无锡，江苏，中国}

摘 要 研究和设计一种融合二代证身份信息的人脸比对识别系统。为了保证系统的注册质量和准确性, 采用验证注册的方式。 在注册阶段, 将摄像机采集图像与二代证中存储的人脸图像进行比对, 将比对结果大于预设阈值的现场图像和对应的二代证上的文字 信息注册入系统。在查询阶段, 将待识别图像与系统数据库中的模板进行比对, 返回前 $\mathrm{N}$ 位相似度较高的人脸照片及对应信息。并且, 支持使用一些辅助身份信息 (例如姓名、身份证号码) 进一步缩小查询结果集。为了提高系统查询准确率, 引入质量评估模块选取高 质量的待识别图像进行人脸查找。实验结果表明, 通过对现场人脸图像进行质量评估以及通讯数据包格式的设计, 可以有效提高该系 统的效率和精度。

关键词 二代证, 人脸识别, 注册, 质量评估, 查询

\section{1. 引言}

目前第二代居民身份证在全国范围内已经基本普及, 由于二代证采用了非接触式 IC 智能芯片来存储身份证唯 一编号、人脸照片和其它基本身份信息, 使得结合二代证 上身份信息的应用逐步推广开来[1]。基于二代证中照片的 人脸识别成为近年来兴起的一种新应用, 具有重要的研究 价值和应用前景[2]。

在注册阶段, 本系统将持卡人现场被采集的人脸图像

中国科学院重点部署项目支持 (资助号: KGZD-EW-102-2)
的生物特征与身份证阅读器所读取的二代证相片的生物特 征进行比对, 从而判定是否为同一个人, 并将通过指定阈 值的现场人脸图像和二代证上的文字信息存入数据库。在 查询阶段, 通过现场采集的人脸图像的生物特征与该数据 库中的生物特征进行比对, 得到库中与该现场采集的人脸 图像最为相似的图像信息以及个人身份信息。系统不仅能 便捷的从二代证获取居民基本信息, 而且还能确保人证合 一和提供身份查询功能。系统可用于车站安检、边境通关 及金融事务办理等公共安全场合 [3], 旨在取代传统的人工 验证及查询方式, 提高验证和查询的效率和准确率。 


\section{2. 系统框架结构及应用功能}

该系统是基于二代证的身份验证注册和身份查询的人 脸识别应用系统, 采用 $\mathrm{C} / \mathrm{S}$ 和 $\mathrm{B} / \mathrm{S}$ 混合架构, 如图 1 所示, 客户端和负载均衡服务器之间采用自定义格式的数据包并 通过基于 TCP 协议的 socket 进行通信。其中客户端通过摄 像机采集的人脸图像和二代证上的照片提供 1:1 验证注册, 通过摄像机采集的人脸图像和一些额外辅助信息（例如姓 名、身份证号) 提供 $1: \mathrm{N}$ 查询功能。负载均衡服务器用来 将客户端请求均衡分配到人脸识别服务器上进行处理。人 脸识别服务器采用集群方式提供人脸生物特征抽取、1:1人 脸特征比对、 $1: \mathrm{N}$ 人脸特征比对、向人脸数据库中增加或 删除人脸记录的服务。系统管理人员可以通过 Web 管理服 务器来管理负载均衡服务器、人脸识别服务器集群及数据 库服务器。数据库服务器主要负责对数据库的操作, 保证 数据库中数据的一致性、完整性以及安全性。

\section{1 客户端}

由一台 PC 机、一台摄像机和一台二代证读卡器组成, 主要提供验证注册和查询两大模块的操作界面。用户可以 通过客户端向服务器提交摄像机实时采集的人脸图像、二 代证照片及其它个人身份信息, 然后根据服务器的响应信 息实时显示相应的验证注册或者查询结果信息。

验证注册模块操作界面如图 2 所示, 二代证读卡器读 取被验证对象的二代证照片及文字信息, 摄像机实时抓取 被验证对象的现场人脸图像。系统首先通过人脸检测算法 对实时捕获的现场图像进行人脸检测 [4], 对无人脸的帧图 像直接丢弃; 然后将二代证上的人脸照片与现场采集的人 脸图像提交到服务器进行人脸 $1: 1$ 比对识别, 如果比对相 似度大于预设阈值, 则将现场照片和二代证上的个人信息 通过人脸识别服务器存入后台数据库中。对于已经存在库 中的人脸对象, 可以重新验证注册并修改身份信息。由于 年龄跨度太大等因素的影响, 相貌的巨大差异使得持证人 无法通过验证, 可以经过管理人员访问 Web 管理服务器, 将该对象的现场人脸照片及个人信息手工录入数据库中。 同时由于二代证上面的照片是通过小波变换压缩存储的低 质量图像[5], 因此把现场实时采集的 $720 p$ (即 $1280 \times 720$ ) 的人脸图像存入人脸数据库, 以保证后期与库中人脸图像 进行 1:N 比对时更加快速和准确。

查询模块操作界面如图 4 所示, 摄像机现场实时捕获 当前对象的人脸照片, 然后经过人脸检测、质量评估、特 征抽取等相关处理后, 与验证注册中所建立的人脸图像特 征库进行 1: $\mathrm{N}$ 比对识别来获取库中前 $\mathrm{N}$ 位相似度较高的人 脸图像信息。本模块还可以通过刷二代证输入或手工录入 当前对象的额外身份信息 (例如姓名、身份证号码), 来进 一步缩小查询范围, 更加准确定位到目标对象。

\section{2 服务器}

本系统主要涉及 4 种不同应用功能的服务器, 分别是 负载均衡服务器、人脸识别服务器集群、数据库服务器和 Web 管理服务器。

负载均衡服务器只进行人脸识别服务器集群的状态收 集、系统一致性维护和负载分配的工作, 并不进行实际的 服务工作, 从而使得服务器集群的结构对用户是透明的。 负载均衡器每隔一定周期 $\mathrm{T}$ (此参数可通过 web 管理服务 器进行配置) 就会收集所有节点的负载信息 (例如: CPU、 内存、硬盘等使用率), 并存入列表中。并且, 定时轮询每 个节点的状态, 更新列表并计算系统平均负载。每个节点 将自身负载与系统设定的阈值做比较, 如果超载则启动自 身的服务器进程, 将任务迁移至轻载的节点。

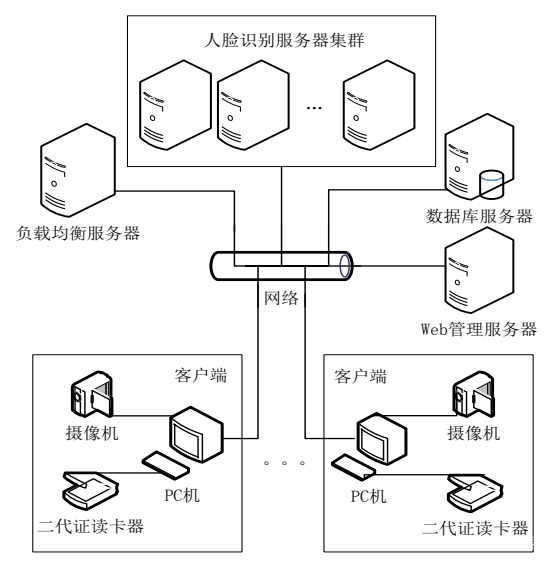

图 1 系统框图

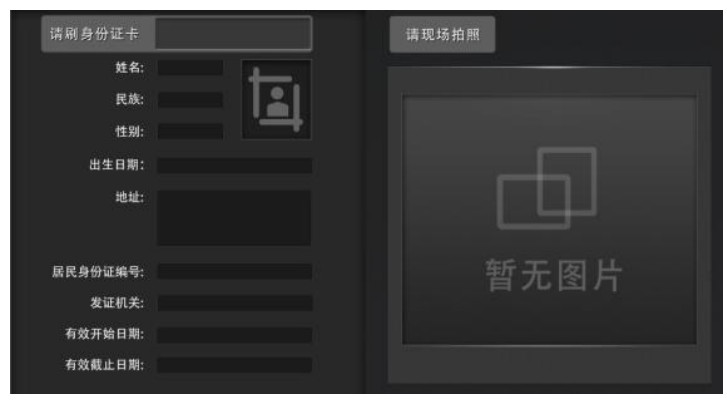

图 2 验证注册操作界面

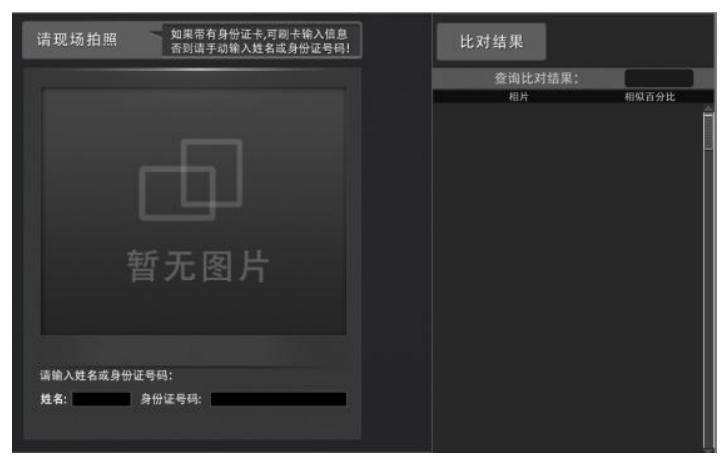

图 3 查询操作界面 
人脸识别服务器是一组真正执行客户请求的服务器, 所提供的服务有人脸特征抽取、 $1: 1$ 人脸特征比对、 $1: \mathrm{N}$ 人 脸特征比对、人脸库中人脸特征数据的增加和删除。其利 用 Linux 虚拟服务器 (Linux Virtual Server) 技术[6]将一组 人脸识别服务器构成一个可伸缩的、高可用网络服务的服 务器集群。该集群中的节点数目是可变的, 当整个系统的 负载超过目前所有节点的处理能力时, 可以很方便地在集 群中增加服务器来满足不断增长的请求负载。每台服务器 拥有相同的内容并提供相同的服务, 当客户请求到达时, 负载均衡服务器会根据负载情况从集群中选出一台服务 器, 将该请求转发到选出的服务器, 并记录这个调度; 当 这个请求的其他报文到达时, 也会被转发到前面选出的服 务器。

数据库服务器主要是接收人脸识别服务器集群和 $\mathrm{Web}$ 管理服务器的数据操作请求, 实现对数据库 (包括人脸图 像表、人脸特征表、个人身份信息表）插入、修改、更新 和查询等功能。其采用基于线程池和数据缓冲区的设计思 想, 在请求到达时首先存入数据缓冲区, 当缓冲区中的数 据准备就绪时, 系统从线程池中轮询某一空闲线程来获取 数据进行处理, 这样消除直接操作数据库及重复创建和销 毁线程带来的延迟, 提高系统响应速度; 而且, 可以通过 web 管理服务器调整线程池中的线程数目, 当请求数目超 过预设阈值时, 强制使其它任何新到的请求一直等待, 直 到获得某个空闲线程来进行处理, 以防资源不足。

Web 管理服务器主要提供管理人员通过 Web 方式查看 和配置负载均衡服务器、人脸识别服务器及数据库服务器 的状态和参数。当合法持证人由于相貌巨大差异等因素无 法通过系统验证注册时, 还可以向数据库提交人脸图像和 其它身份信息等。

\section{3. 质量评估}

在本系统中, 输入图像的质量会影响到后台服务器进 行 1:N 人脸比对识别的效率和准确度。低质量的图片容易 引起系统匹配错误, 降低用户体验。质量评估 [7-8]可以有 效地改善由于输入图像质量过低引起的系统错误, 它可以 对摄像机实时采集的待查询人脸图像的质量进行评估, 如 图 4 所示, 当图像质量高于一定门限时才会被提交到后台 服务器中进行特征抽取和比对识别, 否则图像将被丢弃。 这样方式在某种程度上预防系统错误, 还可以有效的节省 匹配时间。

本系统中通过对人脸图像相关参数进行评估来保证系 统的正确性。参数的详细信息如表 1 所示。当摄像机采集 的现场人脸图像参数达到如表 2 所示的约束时, 该待查询 人脸图像才能符合系统要求, 否则将被直接丢弃。

\section{4. 通讯数据包格式}

为了保证每个客户端节点至负载均衡服务器通讯的稳 定性、可靠性和扩展性, 本系统采用自定义通讯数据格式 的 socket 通信模型。其中负载均衡器上的通信程序负责监 听, 监听地址及端口信息在其配置文件中, 可以通过 web 管理服务器进行更改。每一次连接由客户端节点发起, 并 且每次连接都是短连接。负载均衡服务器对于通讯中的异 常有如下处理: (1) 如果读取包不完整, 超时 30 秒, 则抛 弃该包; (2) 如果是无包头的数据, 直接抛弃。

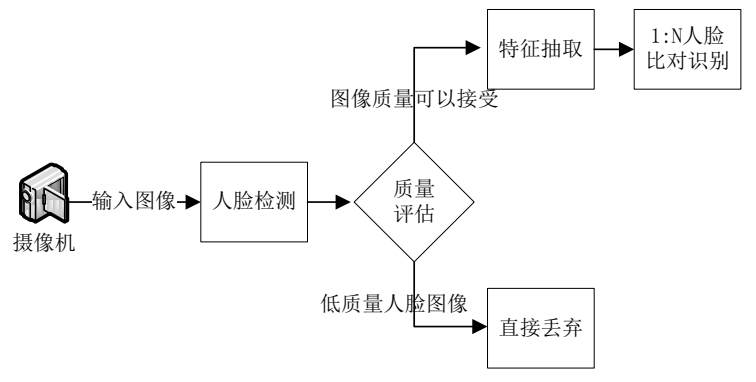

图 4 查询模块中的质量评估结构图

表1 质量评估参数详细信息表

\begin{tabular}{|c|c|c|c|c|}
\hline 参数名称 & 参数类型 & 取值范围 & 系统取值 & 备注 \\
\hline $\begin{array}{c}\text { 两眼最小距离 } \\
\text { (minEyeDistance) }\end{array}$ & float & 正数 & 50 & $\begin{array}{c}\text { 单位是 } \\
\text { 像素 }\end{array}$ \\
\hline $\begin{array}{c}\text { 两眼最大距离 } \\
\text { (maxEyeDistance) }\end{array}$ & float & $\begin{array}{c}\text { 大于最 } \\
\text { 小距离 }\end{array}$ & 10000 & $\begin{array}{c}\text { 单位是 } \\
\text { 像素 }\end{array}$ \\
\hline $\begin{array}{c}\text { 人脸面内 } \\
\text { 旋转角度(roll) }\end{array}$ & float & $0 \sim 180^{\circ}$ & $20^{\circ}$ & $\begin{array}{c}\text { 角度越 } \\
\text { 小越好 }\end{array}$ \\
\hline $\begin{array}{c}\text { 人脸面外旋转角 } \\
\text { 度(frontalAngle) }\end{array}$ & float & $0 \sim 90^{\circ}$ & $45^{\circ}$ & $\begin{array}{c}\text { 角度越 } \\
\text { 小越好 }\end{array}$ \\
\hline $\begin{array}{c}\text { 人脸检测器的置 } \\
\text { 信度(confidence) }\end{array}$ & float & $0 \sim 1$ & 0.7 & $\begin{array}{c}\text { 值越大 } \\
\text { 越好 }\end{array}$ \\
\hline $\begin{array}{c}\text { 人脸质量 } \\
\text { (quality) }\end{array}$ & float & $0 \sim 100$ & 80 & $\begin{array}{c}\text { 值越大 } \\
\text { 越好 }\end{array}$ \\
\hline
\end{tabular}

表2 符合系统要求的人脸图像参数约束表

\begin{tabular}{|c|c|}
\hline 参数名称 & 约束 \\
\hline 两眼距离 & $\begin{array}{c}\text { minEyeDistance } \leq \text { the eye distance } \leq \\
\text { maxEyeDistance }\end{array}$ \\
\hline 人脸面内旋转角度 & $0 \leq$ the roll angle of face $\leq$ roll \\
\hline 人脸面外旋转角度 & $0 \leq$ the frontal angle of face $\leq$ frontalAngle \\
\hline 人脸检测器的置信度 & confidence $\leq$ the confidence of face $\leq 1$ \\
\hline 人脸质量 & quality $\leq$ the quality of face $\leq 100$ \\
\hline
\end{tabular}

通讯中的图像数据包格式如表 3 所示, 图像数据包长 
度包括图像格式和图像数据整体长度。在注册阶段, $1: 1$ 人 脸比对识别中请求数据包格式如表 4 所示; 响应数据包格 式如表 5 所示。在查询阶段, $1: \mathrm{N}$ 人脸比对识别中请求数 据包格式如表 6 所示; 响应数据包格式如表 7 所示，(1) 其中图像 1 至图像 $\mathrm{N}$ 是按照比对结果值从大到小的顺序进 行排序, 即图像 1 是相似度最高的匹配结果, (2) 当 $1: \mathrm{N}$ 查询结果数目为 0 时, 即未查询到符合要求的匹配人脸图 像, 则响应数据包中均不包含图像 1 至图像 $\mathrm{N}$ 的总长度、 数据包及比对结果数据。

表3 图像数据包格式

\begin{tabular}{|c|c|c|}
\hline 参数名称 & 所占字节数 & 备注 \\
\hline 图像格式 & 2 & $\begin{array}{l}\text { BMP:0x0001 ;JPG:0x0002 } \\
\text { PNG:0x0003; 其它:0x0004 }\end{array}$ \\
\hline 图像数据 & 因图像大小而异 & \\
\hline
\end{tabular}

表4 $1: 1$ 人脸比对识别中请求数据包格式

\begin{tabular}{|c|c|c|}
\hline 参数名称 & 所占字节数 & 备注 \\
\hline 包头 & 2 & 0xefef \\
\hline $1: 1$ 比对请求指令 & 1 & 0x01 \\
\hline 二代证照片总长度 & 4 & \\
\hline 现场人脸图像总长度 & 4 & \\
\hline 二代证照片数据包 & 2+照片数据长度 & 见表 3 \\
\hline 现场人脸图像数据包 & 2+图像数据长度 & 见表 3 \\
\hline 包尾 & 2 & 0xfefe \\
\hline
\end{tabular}

表5 $\quad 1: 1$ 人脸比对识别中响应数据包格式

\begin{tabular}{|c|c|c|}
\hline 参数名称 & 所占字节数 & 备注 \\
\hline 包头 & 2 & $0 x e f e f$ \\
\hline $1: 1$ 比对响应指令 & 1 & $0 x 02$ \\
\hline $1: 1$ 比对结果 & 1 & 正常取值范围: $0 \sim 1$ \\
\hline 预设阈值 & 1 & 取值范围: $0 \sim 1$ \\
\hline 包尾 & 2 & 0xfefe \\
\hline
\end{tabular}

表6 $1: \mathrm{N}$ 人脸比对识别中请求数据包格式

\begin{tabular}{|c|c|c|}
\hline 参数名称 & 所占字节数 & 备注 \\
\hline 包头 & 2 & 0xefef \\
\hline $1: \mathrm{N}$ 比对请求指令 & 1 & $0 x 03$ \\
\hline 现场人脸图像总长度 & 4 & \\
\hline 现场人脸图像数据包 & $2+$ 图像数据长度 & 见表 3 \\
\hline 辅助信息标志位 & 1 & $\begin{array}{c}\text { 0:身份证号码; } 1 \text { :姓名; } \\
\text { 2: 以上两个字段皆有 }\end{array}$ \\
\hline 辅助信息字段 & 100 & 姓名、身份证号码 \\
\hline 包尾 & 2 & 0xfefe \\
\hline
\end{tabular}

表7 1:N人脸比对识别中响应数据包格式

\begin{tabular}{|c|c|c|}
\hline 参数名称 & 所占字节数 & 备注 \\
\hline 包头 & 2 & Oxefef \\
\hline 1: $\mathrm{N}$ 比对响应指令 & 1 & 0x04 \\
\hline 1: $\mathrm{N}$ 查询结果数目 & 1 & \\
\hline 匹配图像 1 总长度 & 4 & \\
\hline 匹配图像 1 数据包 & $2+$ 图像 1 数据长度 & 见表 3 \\
\hline $\begin{array}{l}\text { 待查询现场图像与匹 } \\
\text { 配图像 } 1 \text { 的比对结果 }\end{array}$ & 1 & $\begin{array}{l}\text { 正常 取 值范 } \\
\text { 围: } 0 \sim 1\end{array}$ \\
\hline \multicolumn{3}{|c|}{$\ldots$} \\
\hline 匹配图像 $\mathrm{N}$ 总长度 & 4 & \\
\hline 匹配图像 N 数据包 & 2+图像 $\mathrm{N}$ 数据长度 & 见表 3 \\
\hline $\begin{array}{l}\text { 待查询现场图像与匹 } \\
\text { 配图像 } \mathrm{N} \text { 的比对结果 }\end{array}$ & 1 & $\begin{array}{l}\text { 正 常 取 值 范 } \\
\text { 围: } 0 \sim 1\end{array}$ \\
\hline 包尾 & 2 & Oxfefe \\
\hline
\end{tabular}

\section{5. 实验结果}

在这部分中, 通过一系列实验来测试本系统的性能。 其中有一个注册集, 包含 21 个人的二代证照片和现场人脸 图像; 两个测试集, 一个是原始采集的 22 个人共 545 张现 场人脸图像, 图像大小为 $1280 \times 720$, 含有模糊、姿态、光 照及表情等多种因素的影响, 另一个为在原始采集的基础 上通过质量评估模块过滤后, 符合系统要求的 22 个人共 502 张现场人脸图像。最终得到的 ROC 曲线如图 5 所示。

可以看到, 在一定的错误接受范围内, 当有质量评估 模块时, 系统正确识别率明显提高。当系统的错误接受率 为 $10 \%$ 时, 含有质量评估模块的系统正确识别率可以达到 95\%，这样的结果已经达到预期效果。

对现场采集的人脸图像进行质量评估, 部分示例如图 6 所示。其中如图 6 (a) 所示, 是满足系统要求的现场人 脸图像; 如图 6 (b) 所示, 由于模糊、姿态、光照和表情 的多种因素影响, 导致所示的人脸图像不符合系统的要求。

系统各组件的软硬件参数如表 8 所示, 本文对系统各 部分及整体运行效率也进行了相关测试。其中 PC 机对每 一张二代证照片和现场采集的 720p 图像 (图像中只包含一 张人脸的情况下) 分别进行人脸检测操作, 平均耗时分别 为 $74.34 \mathrm{~ms} 、 20.21 \mathrm{~ms}$; 服务器对每一张二代证照片和现场 采集的 720p 图像 (图像中只包含一张人脸的情况下) 分别 进行人脸特征提取操作, 平均耗时分别为 $130.07 \mathrm{~ms}$ 、 $70.51 \mathrm{~ms}$; 服务器进行 $1: 1$ 人脸比对识别和 $1: \mathrm{N}$ 人脸比对识 别, 平均速度分别为 2.57 微秒, 400 万/秒。在此基础上, 并且假设网络通信正常的情况下, 当进行验证注册时, 从 客户端提交请求数据包到显示服务器的响应结果, 平均耗 
时大约为 $0.71 \mathrm{~s}$; 当进行查询验证时, 针对 21 个注册集, 从客户端提交请求数据包到显示服务器的响应结果, 平均 耗时大约为 $0.96 \mathrm{~s}$ 。

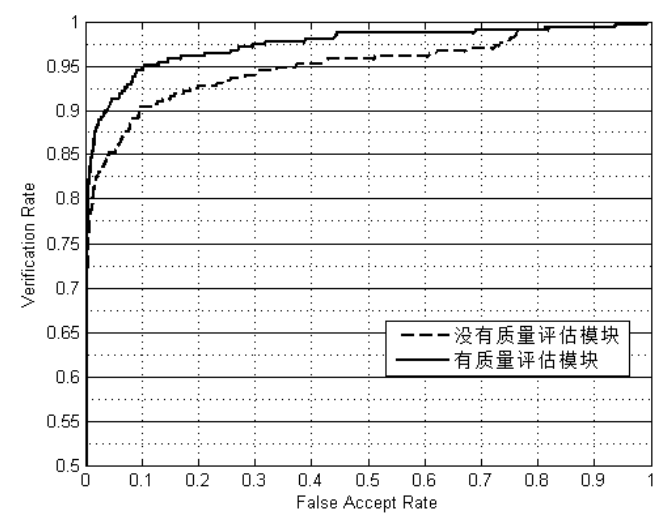

图 5 系统 ROC 曲线

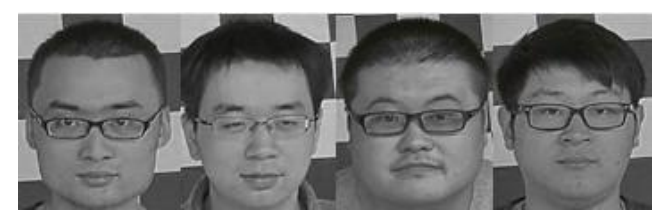

(a) 满足系统要求的现场人脸图像

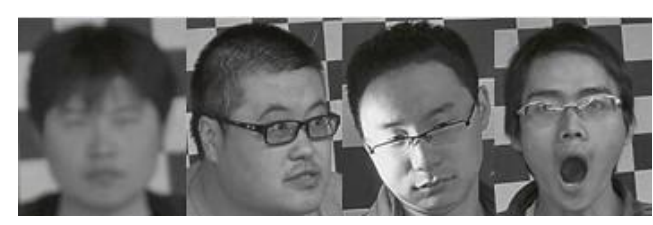

(b) 不符合系统要求的现场人脸图像

图 6 质量评估结果的部分示例

表 8 系统各组件的软硬件参数

\begin{tabular}{|l|l|}
\hline 组件名称 & 软硬件参数配置 \\
\hline 二代证读卡器 & $\begin{array}{l}\text { 国腾实业-联机型 GTICR100-01 } \\
\text { 读卡时间: } \leq 1.0 \mathrm{~s}\end{array}$ \\
\hline 摄像机 & $\begin{array}{l}\text { 海康摄像机 DS-2CD864FWD-E } \\
\text { 采集帧率: } 25 \text { 帧/秒 }\end{array}$ \\
\hline \multirow{3}{*}{ PC 机 } & $\begin{array}{l}\text { CPU : Intel i3 3220 3.4GHz } \\
\text { 内存: 4GB } \\
\text { 系统: win7 (32 位) }\end{array}$ \\
\hline 服务器 & $\begin{array}{l}\text { CPU : Intel Xeon E5645 2.4GHz } \\
\text { 内存: 128GB } \\
\text { 系统: RedHat Enterprise Linux 5 (64 位) }\end{array}$ \\
\hline
\end{tabular}

\section{6. 结语}

本文基于二代证身份信息提出一种结合人脸图像质量 评估的人脸识别系统。该系统不仅提供验证注册功能, 还 提供查询功能。正如上面所提到的那样, 由于二代证照片
图像质量低, 为了提高查询时人脸比对速度和准确度, 本 系统将现场采集的人脸图像注册入库组成人脸图像库; 由 于年龄跨度大导致验证无法通过时, 管理人员可以通过 web 方式手工注册至数据库中; 为了保证通信效率和通信 数据的完整性、一直性及安全性, 本系统对客户端和负载 均衡器之间的通信数据包格式进行了自定义设计。为了保 证待查询人脸图像的质量, 本系统提供质量评估模块以滤 掉质量差的图像。实验结果表明当错误接受率为 $10 \%$ 时, 含有质量评估模块的系统正确识别率高达 $95 \%$, 具有良好 的实际应用效果。

在今后的工作中, 将进一步优化质量评估参数、改进 通讯数据包格式的设计, 来提升系统的泛化能力和性能。

\section{参考文献 (References)}

[1] Tie-jun Pan, Chun-lei Xiong, Lei-na Zheng, et a1. ONE-CARD System Based on the Second Generation ID Card in China[C] //Proceedings of the International Conference on E-Business and E-Government. China: Guangzhou, 2010:108-111.

[2] Ibrahim, R., Zin, Z.M.. Study of automated face recognition system for office door access control application. // 2011 IEEE 3rd International Conference on Communication Software and Networks (ICCSN). China: Xi'an, 2011:132-136.

[3] Stan Z. Li. Face Recognition-Analysis of security biometrics applications and market development. China Security \& Protection, 2013(5):63-66.

[4] Qiong Liu, Guang-zheng Peng. An improved adaboost face detection algorithm. Computer Applications and Software, 2011, 28(3):265-268.

[5] Feng-yi Shen. Image Processing and its Implementation in Second ID Card. Shanghai: Shanghai Jiao Tong University, 2007.

[6] Inhyuk Kim, Taehyoung Kim, Young Ik Eom. NHVM: Design and Implementation of Linux Server Virtual Machine Using Hybrid Virtualization Technology //Proceedings of the International Conference on Computational Science and Its Application. Fukuoka, 2010:171-175.

[7] Fei Yang, Jian-bo Su, Jing-wen Dai.Fast quality assessment of face images for face recognition. // Proceedings of the 27th Chinese Control Conference. China: Kunming, 2008:531-535.

[8] Bharadwaj, S., Bhatt, H., Vatsa, M., et a1. Quality assessment based denoising to improve face recognition performance //Proceedings of the IEEE Computer Society Conference on Computer Vision and Pattern Recognition Workshops. Colorado Springs, 2011:140-145. 